\title{
Diseño de una aplicación de realidad aumentada para uso docente
}

\section{Gabriel Peris $^{\mathrm{a}}$, Ana Iris Escudero ${ }^{\mathrm{b}}$ y Begoña Peña ${ }^{\mathrm{c}}$}

Universidad de Zaragoza - Departamento de Ingeniería Mecánica (España) e-mail: agabcses@gmail.com, baniresc@posta.unizar.es, ${ }^{\mathrm{c}}$ bpp@unizar.es

\begin{abstract}
Augmented reality technologies have great potential in many sectors and, in particular, in the field of education. In order to reinforce learning process in a more attractive fashion for students, increasing the engagement in the subject, this paper presents the procedure followed in the preliminary design of an application of augmented reality for learning purposes.Specifically, the augmented reality will be used to show the operation of a drinking fountain based on the vapor compression refrigeration cycle. The APP would be useful for subjects in the field of Thermal Engineering in Engineering studies.In this work, a review of the state of the art and associated emerging technologies has been presented, as well as the results of the surveys carried out on teachers and students. The procedure followed in the development of the APP is also explained and the preliminary design is shown.
\end{abstract}

Keywords: Augmented reality, Active learning, Mobil devices,Higher Education Innovation, Engineering Thermodynamics.

\section{Resumen}

Las tecnologías de realidad aumentada presentan un gran potencial en muchos sectores $y$, en particular, en el ámbito de la educación. Con el fin de reforzar el aprendizaje de una forma más atractiva para los alumnos, que fomente el seguimiento de las asignaturas, en este trabajo se presenta el procedimiento seguido en el diseño preliminar de una APP con fines docentes.Concretamente, se pretende utilizar la realidad aumentada para mostrar el funcionamiento de una fuente de agua fría, basada en el ciclo de refrigeración por compresión de vapor. La APP sería útil para asignaturas del campo de la Ingeniería Térmica en estudios de Ingeniería. En este trabajo, se ha presenta la revisión del estado del arte y de las tecnologías emergentes asociadas, asi como los resultados de las encuestas realizados a profesores y estudiantes. También se explica el procedimiento seguido en el desarrollo de la APP y se muestra el diseño preliminar .

Palabras clave: Realidad aumentada, Metodologías activas, Dispositivos móviles, Docencia Universitaria, Innovación, Termodinámica.

\section{Introducción}

Las actividades de laboratorio y prácticas han demostrado jugar un rol importante en proveer a los alumnos de experiencias educativas valiosas. Las prácticas de laboratorio, además de la reforzar los conceptos introducidos mediante los métodos teóricos tradicionales, son particularmente efectivas a la hora de promover la colaboración y las habilidades de comunicación social, las cuales son destrezas importantes que deben adquirir los graduados, tanto como una base teórica rigurosa. 
Sin embargo, puede ocurrir que, en ocasiones, los fondos destinados a la experimentación sean más limitados y en consecuencia se vea reducido el acceso a las instalaciones y, por tanto, inhibida la calidad de este tipo de actividad. Para muchas instituciones educativas, los recursos limitados hacen que mantener actualizadas las instalaciones de laboratorio sea una carga económica importante. Debido a que nos encontramos en un panorama tecnológico que evoluciona muy rápidamente, el equipo utilizado en los laboratorios y prácticas, ya no está a la altura en muchas ocasiones de las expectativas de los estudiantes. Además, en muchas zonas del mundo donde hay falta de infraestructuras, educadores y fondos económicos, el acceso a la educación de calidad simplemente es algo inaccesible. Por otra parte, en muchas ocasiones los alumnos tienen que invertir mucho tiempo en poner a punto las instalaciones, calibrándolas o resolviendo problemas en los equipos, en detrimento del tiempo dedicado a la propia experimentación en sí (Frank, 2017). Alumnos que, además, se pueden considerar como "nativos digitales", porque en su vida cotidiana están interactuando constantemente con mucha información gráfica que proviene de videojuegos, internet o películas 3D.

Para abordar todos estos desafíos han surgido nuevas soluciones tecnológicas como laboratorios virtuales, que permiten a los alumnos interactuar con experimentos simulados desde cualquier lugar y en cualquier momento (Maiti, 2012). Durante la pasada década se han extendido las Tecnologías de Comunicación e Información (TIC) en todos los ámbitos de la sociedad. Estas tecnologías se presentan como un conjunto de herramientas apropiadas para el contexto social actual, en el que la necesidad de acceso a la información en cualquier momento y lugar, así como los rápidos cambios tecnológicos y la demanda de una educación de alto nivel (que requiere de una constante actualización) representan las principales características (Martín Gutierrez, 2015). Así pues, durante los últimos años, instituciones educativas de todos los niveles han intentado evolucionar mediante la integración y el uso de las TIC en las metodologías de enseñanza (Dede, 2000).

En esta línea, muchas universidades han adaptado ya entornos de aprendizaje virtuales con el objetivo de mejorar los procesos de enseñanza y muchos investigadores, profesores y pedagogos se han centrado en nuevos métodos de visualización. Por ejemplo, Pan et. al. (Pan, 2006) demostraron que el aprendizaje virtual puede proporcionar las herramientas adecuadas que permitan a los usuarios aprender de una manera rápida y eficaz, interactuando con entornos virtuales. El interés de los educadores en estas tecnologías radica principalmente en la intención de obtener un mayor compromiso y un incremento en la motivación del estudiante por entender el contenido, lo que permite guiar a los alumnos hacia una mejora de los resultados académicos (Krejins, 2013, Roca, 2008, Shen, 2013). Son numerosos los estudios que han abordado las cuestiones, problemas y soluciones para la innovación educativa basada en las TICs (Guilarte Martín Calero, 2008) y muchas las prácticas educacionales comparativas y pruebas llevadas a cabo con el fin de definir los efectos que implica incorporar estas metodologías en los procesos de enseñanza-aprendizaje (Law, 2008).

Algunas de las aplicaciones educativas más útiles han sido aquellas basadas en técnicas de visualización 3D que permiten proporcionar experiencias de aprendizaje visualmente atractivas. Hay dos principales categorías de visualización 3D (Dong, 2013): la Realidad Aumentada (AR) y la Realidad Virtual (VR). Mientras que la tecnología RV sumerge completamente al usuario en un entorno sintético en el que puede interactuar sin percibir el mundo real, la tecnología AR, presentada por primera vez por Milgram y Takemura (Milgram, 1994), permite al usuario ver el mundo real combinado con objetos virtuales generados por ordenador (como texto, imágenes, video, modelos o animaciones 3D) superpuestos o fusionados con el entorno real. Según Azuma (Azuma, 1997), la realidad aumentada permite mejorar la percepción sensorial que tiene el usuario del mundo real añadiendo una capa contextual de información. 
La realidad aumentada es una de las tecnologías 3D más utilizadas y se está aplicando en muchos campos de la sociedad: medicina, arquitectura, marketing, publicidad, militar, arqueología, ocio, etc. (Craig, 2013). Algunos investigadores han señalado que la AR tiene posibilidades educativas potenciales que son especialmente útiles en los campos de la ciencia, la tecnología, la ingeniería y las matemáticas (su acrónimo en inglés, STEM), fomentando la capacidad espacial, las habilidades prácticas, la comprensión conceptual y el aprendizaje en la investigación científica (Bujak, 2013, Cheng, 2013, Dunleavy, 2009, Wu, 2013, Dong, 2013, Ibáñez, 2018).

Además, se ha demostrado que las principales características de los medios de AR, como son la inmersión sensorial, la navegación y la manipulación, parecen funcionar como promotores de emociones positivas, a la vez que los alumnos aprenden, y mejoran los resultados de aprendizaje de una manera más eficiente (Cheng, 2013, Wu, 2013). Radu (Radu, 2014) concluye que la AR es útil para aumentar la motivación de los estudiantes, fomentar la colaboración entre los estudiantes, desarrollar habilidades espaciales y mejorar el rendimiento en las tareas físicas. Akçayır and Akçayır (Akçayir, 2017) reportó un aumento en el número de estudios de AR y concluyó que la tecnología podría tener el potencial de apoyar el aprendizaje y la enseñanza cuando se daban situaciones como necesidad de más tiempo de clase o grupos excesivamente numerosos.

Bacca et. al. (Bacca, 2014) afirman que las principales ventajas de la AR son la motivación, la interacción y la colaboración, mientras que las principales limitaciones se deben a los problemas que puede generar el desconocimiento acerca de cómo usar la tecnología. Sin embargo, una de las ventajas más significativas del AR es que permite el aprendizaje autónomo y colaborativo por igual y es precisamente ahí donde radica el mayor potencial de la tecnología (Billinghurst, 2002, Kaufmann, 2003, Martín Gutiérrez, 2015). En particular, resultan de interés las aplicaciones que se han desarrollado para aprovechar los dispositivos personales de los estudiantes en herramientas de asistencia para trabajar en laboratorios de ciencia e ingeniería (Frank, 2017, Williams, 2011, Nguyen, 2015, Fonseca, 2014). Además, los dispositivos móviles, smartphones particulares son plataformas ideales para la tecnología AR.

La realidad aumentada es, pues, una tecnología prometedora en el ámbito de la educación para aumentar la motivación y facilitar la aplicación de conocimientos teóricos en entornos prácticos. La posibilidad de utilizar para ello dispositivos móviles, que están al alcance de la mayoría de estudiantes, hace de estas tecnologías una apuesta muy interesante. En este artículo se presenta el diseño de una aplicación de realidad aumentada que tiene como objetivo presentar a los estudiantes de ingeniería el funcionamiento de un ciclo de refrigeración para el enfriamiento de agua en las fuentes que habitualmente se encuentran en muchos edificios públicos, entre ellos la propia Universidad de Zaragoza. La aplicación persigue que los estudiantes tomen conciencia de que los conceptos adquiridos en las clases teóricas tienen utilidad en sistemas de uso cotidiano, así como fomentar el interés por la disciplina de la termodinámica.

\section{Objetivos}

El trabajo aquí presentado ha tenido como objetivos principales el diseño y el desarrollo de una aplicación de realidad aumentada para dispositivos móviles con fines educativos. Concretamente, en esta ponencia se presentan las primeras fases del desarrollo de esta aplicación:

- el estudio previo realizado sobre la evolución de las tecnologías AR y sobre las herramientas actuales de hardware y software para el desarrollo de aplicaciones de este tipo,

- las encuestas realizadas a profesores y estudiantes sobre su percepción de las tecnologías AR,

- la selección de la asignatura objetivo y de los contenidos formativos,

- el diseño preliminar de una aplicación dirigida a estudiantes de ingeniería. 


\section{Desarrollo de la innovación}

El procedimiento seguido para el diseño preliminar de la aplicación de realidad aumentada se resume en el diagrama de la Figura 1. Por un lado, se ha realizado la revisión del estado del arte, en cuanto a tecnologías de hardware y de software. Por otro lado, se han desarrollado encuestas en Google Forms para estudiantes, que se han distribuido a través de los cursos de varias asignaturas en la plataforma Moodle, y para profesores de la Escuela de Ingeniería y Arquitectura de la Universidad de Zaragoza.

A partir de este trabajo previo se han seleccionado las herramientas más adecuadas para el desarrollo de la aplicación y se ha procedido al diseño de pantallas tipo. Los contenidos formativos que se quieren trabajar se tienen en cuenta para la concepturalización de la estructura de la APP y de los flujos principales para la navegación del usuario. A continuación se se procede a elaborar los wireframes, realizar el diseño virtual para finalmente pasar a la fase de desarrollo.

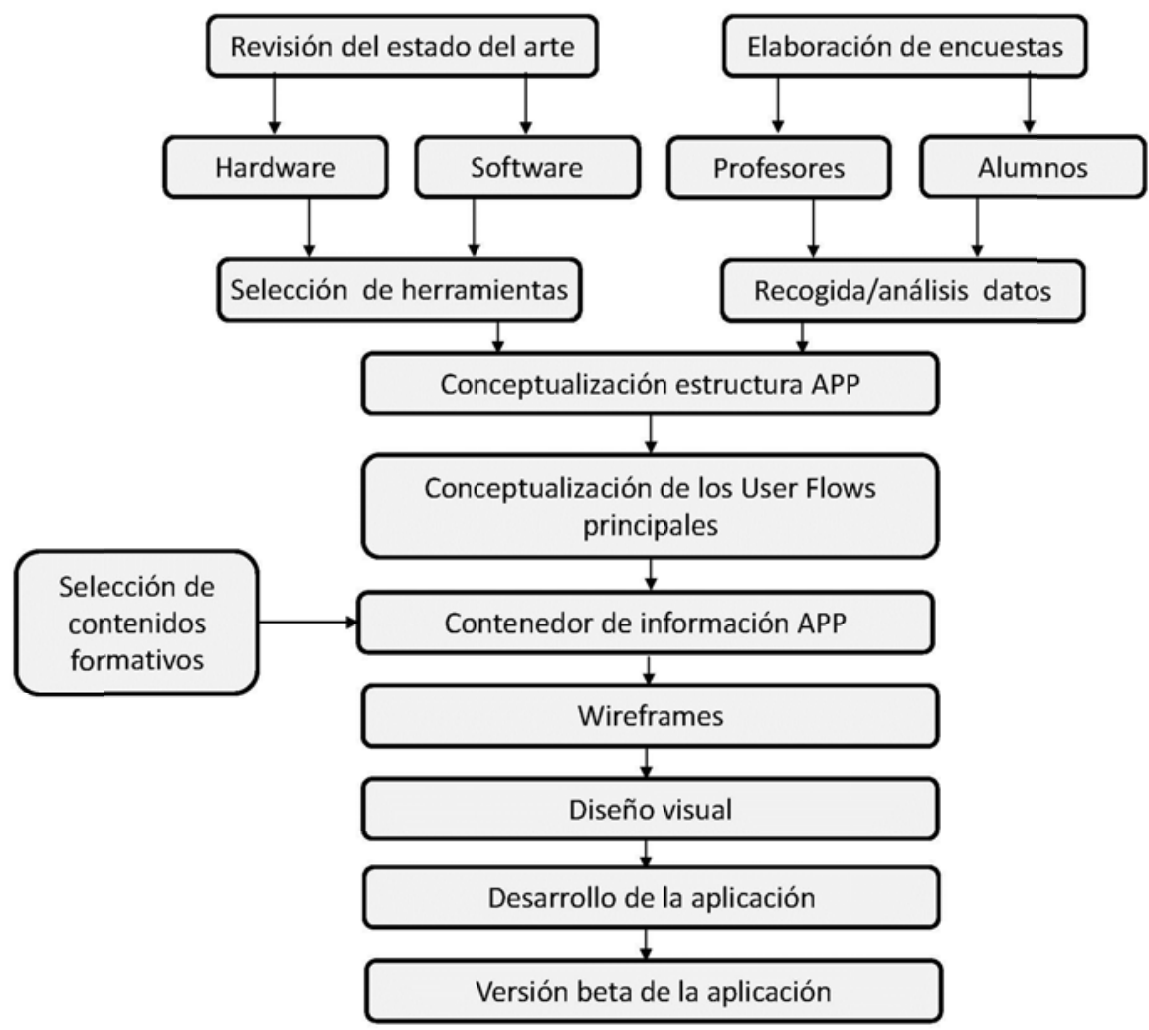

Figura 1. Fases del desarrollo de la aplicación

\subsection{Revisión de herramientas actuales}

A día de hoy existen multitud de dispositivos capaces de analizar su posición y el entorno, comprenderlo y mostrar imágenes sobre el mismo. En realidad las únicas características necesarias para un dispositivo de AR son una pantalla, una cámara y un set de sensores que permita al dispositivo conocer su posición y orientación. Los cálculos computacionales pueden realizarse en el propio aparato o ser enviados a una computadora o servidor externo de mayor potencia.

En cuanto a los dispositivos para Realidad Aumentada existen tanto productos especialmente diseñados para ello (Microsoft Hololens, Magic Leap), como desarrollos en smartphones, siendo estos últimos los más comunes entre el gran público. Desde sus comienzos, Hololens ha sido un producto orientado al 
mundo profesional (HoloLens, 2020). Se trata de unas gafas de grandes dimensiones, en las que el HUD ocupa toda la superficie del cristal. Microsoft ha desarrollado una gran cantidad de aplicaciones para el dispositivo, desde videollamadas con todas las personas presentes en una sala virtual hasta el diseño en el mundo real con herramientas de CAD en AR. El gran problema de esta tecnología es la gran potencia de procesamiento que necesita, por lo que ha de hacer uso de ordenadores de alta gama.

Magic Leap es de uno de los recién llegados (MagicLeap, 2020). Sobre el papel, promete una potencia de procesamiento y una sensación de inmersión superior a sus competidores. Esto es debido a que la potencia de procesamiento se encuentra dentro del propio dispositivo, sin depender de ordenadores externos. Sin embargo, el usuario debe cargar con un mini ordenador colgado de su cadera, con lo que la experiencia no es del todo ideal. Además, la potencia de computación no es ni de lejos comparable a la que cabría esperar de un ordenador tope de gama conectado de forma inalámbrica a otros dispositivos como el Hololens.

En cuanto a los teléfonos móviles, el segmento de la AR para smartphones ha experimentado un crecimiento exponencial en los últimos años. Los dispositivos que cargamos en los bolsillos son cada vez más potentes, ya no solo en capacidad de computación, sino también en lo referente a la precisión de sus sensores, las baterías y las pantallas. Algunos como el nuevo iPad Pro (2020) ya comienzan a introducir hardware específico para la detección de la profundidad en el entorno, el sensor LIDAR (LIDAR, 2020).

Con respecto al desarrollo de software, muchas empresas como Google se han lanzado ya a la creación de SDKs que permitan la estandarización del software y el desarrollo de la tecnología. Apple también ha realizado esfuerzos en este campo, aunque dado lo restrictivo de su ecosistema cuenta con un software más limitado. Para contrarrestarlo y atraer desarrolladores está haciendo uso de nuevas implementaciones en su hardware, como el sensor LIDAR del que hablábamos anteriormente. Si hacemors uso de un enfoque más genérico, ya no centrado únicamente en los smartphones, podemos hablar de otras muchas herramientas y sistemas para construir experiencias de AR, como son Unity, Vuforia (ahora parte de Unity), Kudan o Spark AR Studio.

ARCore es la plataforma de Google desarrollada para la creación de aplicaciones y experiencias en realidad aumentada (ARCore, 2020). Está formada por un conjunto de APIs que permiten incluso compartir dichas experiencias entre terminales de Android e iOS. En concreto, ARCore dota a los terminales de herramientas de software capaces de detectar movimiento del terminal y de su entorno, entenderlo, y estimar la iluminación correcta de los objetos virtuales que se sitúan en él. Desde el 2017 Google ha abandonado el desarrollo de hardware AR para centrarse en ARCore (Google, 2020).

\subsection{Encuestas y entrevistas}

Se han desarrollado dos encuestas específicas para profesores y alumnos, respectivamente, con cuatro bloques: un primer bloque con preguntas generales para situar el contexto del encuestado, un bloque de preguntas sobre la experiencia en tecnologías digitales básicas, un tercer bloque sobre el uso de las TIC con fines docentes y un último bloque de preguntas sobre las tecnologías AR y VR. Las dos encuestas, implementadas en Google Forms, comparten 11 preguntas. En la siguiente tabla se resume la temática de las preguntas y el número de preguntas por bloque.

Tabla 1. Distribución de preguntas en las encuestas por temática

\begin{tabular}{ccccc}
\hline Público objetivo & Contexto & Experiencia & TIC & AR / VR \\
\hline Profesorado & 4 & 4 & 2 & 7 \\
Alumnado & 6 & 5 & 2 & 7 \\
\hline
\end{tabular}

(c) B BY-NC-ND 2020, Universitat Politècnica de València

Congreso In-Red (2020) 


\subsection{Selección de contenidos formativos}

La base de la aplicación a desarrollar sirve a priori para cualquier materia si se seleccionan adecuadamente los contenidos y los elementos virtuales. Para el trabajo aquí presentado, el diseño se ha orientado al Área de Máquinas y Motores Térmicos, más concretamente hacia el aprendizaje de los ciclos de refrigeración por compresión de vapor. En particular, la realidad aumentada se aplica a una típica fuente en la que, mediante este tipo de ciclos de refrigeración, se enfría el agua (Figura 1).

Los contenidos a tratar se agrupan en cinco actividades dentro de la aplicación:

- ¿Para qué sirve un ciclo de refrigeración?

- Descripción e identificación de equipos y parámetros de funcionamiento del ciclo

- Funcionamiento termodinámico

- Análisis termodinámico

- Evaluación o autoevaluación

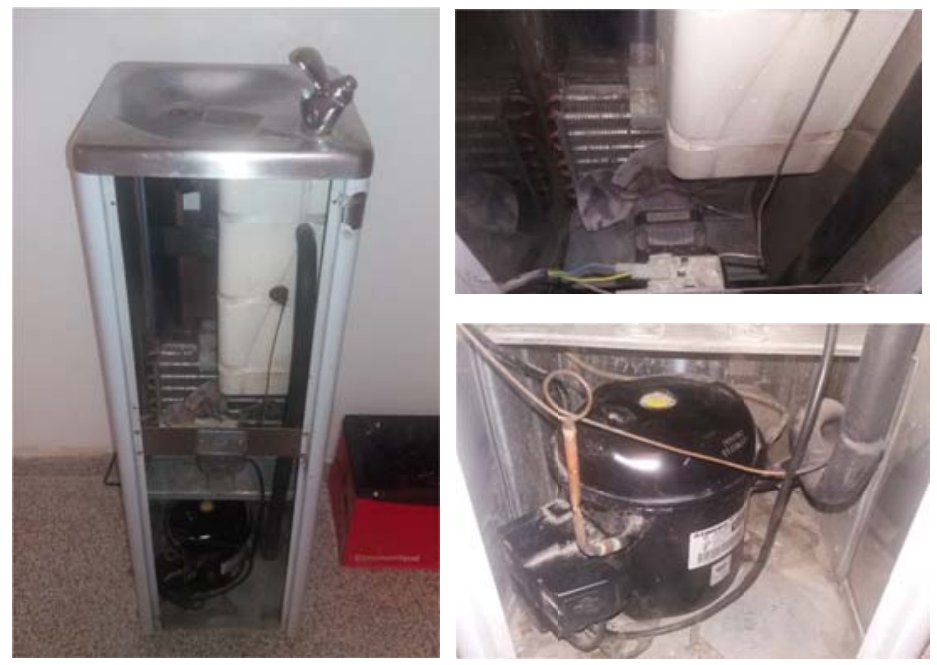

Figura 1 Instalación térmica objeto central de la aplicación de AR.

\section{Resultados}

\subsection{Encuestas a los estudiantes}

Las encuestas elaboradas en Google Forms se han distribuido entre los estudiantes de cinco grados de la Escuela de Ingeniería y Arquitectura a través de los cursos Moodle de diferentes asignaturas. Si bien la participación ha sido baja, se han podido obtener algunos resultados interesantes. Los principales se resumen a continuación:

- Las actividades de enseñanza preferidas se refieren a visitas a instalaciones u obras, o a prácticas que conlleven manipulación manual de instrumentos o herramientas e requieran cierta creatividad.

- Un $72 \%$ de los estudiantes que participaron ha tenido alguna experiencia de AR/VR. En todos los casos, muestran un alto grado de satisfacción, considerándolas atractivas, aunque no diferencian claramente ambas tecnolgías. Un 30\% indicaron sensación de mareo. 
- $\quad$ Sobre el uso de herramientas TIC en sus clases universitarias, el $61 \%$ respondieron que rara vez o nunca han sido utilizadas. Entre los que sí que han utilizado herramientas de este tipo, Kahoot y Socrative son las más populares.

- Sobre el futuro de la tecnología AR, todos opinan que es prometedora y que tiene potencial en todos los sectores. Casi el 50\%, considera además que en los próximos años se aplicará en la vida cotidiana y para el ocio.

- En cuanto al uso de AR en la enseñanza, el 95\% se muestra entusiasmado con su aplicación en docencia, aunque algunos señalan que sólo resultaría útil si los usuarios las utilizan correctamente y están bien diseñadas.

- Sobre la finalidad de las actividades de AR, el 95\% coincide en que sería útil para mostrar situaciones inaccesibles en la vida real, mientras que tan sólo el $10 \%$ considera que podría fomentar el aprendizaje autónomo. Mejorar la compresión de conceptos, fomentar la curiosidad y la asistencia a clase son elegidas en menor medida, concretamente, por el $66 \%, 61 \%$ y el $55 \%$, respectivamente.

- Como posibles usos apuntan principalmente a la visualización de máquinas en operación, visitas a obras, visualización de piezas $3 \mathrm{D}$, testear diseños en entornos reales y a la realización de experimentos virtuales.

\subsection{Encuestas a los profesores}

Las encuestas también se han elaborado en Google Forms y se han distribuido a través del correo electrónico entre profesores de tres áreas de conocimiento: Máquinas y Motores Térmicos, Expresión Gráfica en la Ingeniería e Ingeniería de Sistemas y Automática. A pesar de la baja tasa de respuestas, se puede extraer algunas conclusiones interesantes:

- El 57\% de los profesores reconocen no utilizar ningún tipo de herramienta TIC en la docencia, lo que es coherente con la respuesta de los estudiantes. Entre los que sí que las utilizan, destacan Kahoot y Socrative.

- El porcentaje de profesores con alguna experiencia en AR o VR es algo menor que entre los alumnos: $57 \%$. En cuanto a al uso de la tecnología AR en el futuro, reconocen su potencial pero todos se muestran mucho más cautos sobre su expansión.

- En cuanto a su aplicación con fines docentes, consideran que puede ser útil, pero se muestran más cautos que los estudiantes. Más del $80 \%$ inciden en que debe usarse puntualmente, "sin abusar".

- Sobre el uso docente de estas tecnologías, el $67 \%$ se sentirían cómodos, mientras que el resto responden que "tal vez".

- En cuanto a la finalidad de utilizar AR en docencia, más del $80 \%$ coinciden con los estudiantes en que sería principalmente útil para mostrar situaciones o elementos inaccesibles en la vida real y que no es útil para fomentar el aprendizaje autónomo. Mejorar la comprensión de conceptos y fomentar curiosidad son elegidas por un $65 \%$. Su opinión en cuanto a fomentar la asistencia a clase es menos optimista que entre los estudiantes (15\%).

- En cuanto a las actividades docentes de AR, las propuestas son similares a las de los estudiantes: realizar visitas virtuales, evitar actividades con riesgo, captar la atención en clase y mostrar situaciones o el funcionamiento de equipos que en la vida real no pueden verse. 


\subsection{Diseño de la aplicación}

El diseño de la APP incluye varias fases: conceptualización de la estructura de la APP, conceptualización del flujo de navegación del usuario (user flow), inclusión de los contenidos formativos en la APP, diseño de las pantallas tipo (wireframes) según su funcionalidad, diseño visual y finalmente desarrollo de una primera versión de la APP.

La Figura 2 muestra el user flow: tras la pantalla de inicio, el usuario se registra y selecciona la actividad que quiere realizar. Tras ello, el usuario navega por diferentes pantallas: de explicación, de evaluación o autoevaluación (quizz) y de realidad virtual interactuando con el equipo real (la fuente en este caso).

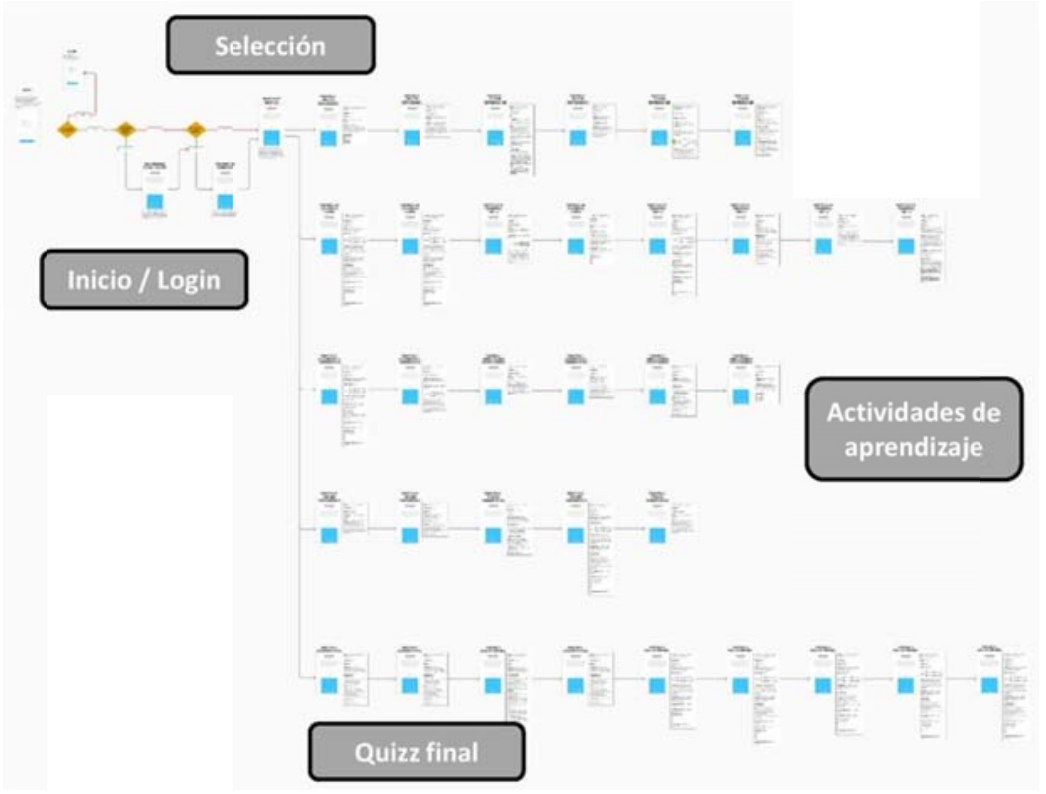

Figura 2.Concepto general de los flujos de navegación del usuario.

\section{Conclusiones}

En este trabajo se ha presentado el procedimiento seguido para el diseño de una APP para dispositivos móviles con realidad aumentada con fines educativos. Las encuestas realizadas muestran un mayor entusiasmo por parte de los alumnos que por parte de los profesores, si bien coinciden en destacar su potencial y su utilidad para mostrar situaciones o equipos inaccesibles en la vida real. Ambos coinciden también en no considerar esta tecnología de utilidad para el aprendizaje autónomo.

En cuanto al diseño de la aplicación, se ha definido el caso de estudio y los contenidos formativos, quedando estructurados en 5 actividades. La primera versión funcional de la APP estará disponible en los próximos meses y se espera validarla en el semestre de otoño del curso 2020-2021 con los estudiantes de Termodinámica Técnica del Grado en Ingeniería en Tecnologías Industriales.

\section{Agradecimientos}

Este trabajo se ha desarrollado dentro del proyecto de innovación docente PIIDUZ_19_265 (Programa de Proyectos de Innovación Docente para Grupos de Profesores del Vicerrectorado de Política Académica de la Universidad de Zaragoza). 


\section{Referencias}

AKÇAYIR, M., AKÇAYIR, G. (2017). "Advantages and challenges associated with augmented reality for education: A systematic review of the literature" en Educational Research Review, vol. 20, p. 1 -11.

AZUMA, R. T. (1997). “A survey of augmented reality” en Presence: Teleoperators and Virtual Environments, vol. 6, p. 355-385.

ARCORE (2020). ARCore overview. Documentación de Google. $<$ https://developers.google.com/ar/discover>. [Consulta : 22 de marzo de 2020].

BACCA, J., BALDIRIS, S., FABREGAT, R., GRAF, S. (2014). "Augmented reality trends in education: A systematic review of research and applications" en Journal of Educational Technology \& Society, vol. 17, issue 4, p. 133.

BILLINGHURST, M., KATO, H. (2002). "Collaborative augmented reality" en Proceedings of Communications of the ACM, vol. 45, issue 7, p. 64-70.

BILlinghuRST, M., KATO, H., POUPYREV, I. (2011). "The MagicBook - Moving seamlessly between reality and virtuality" en Computer Graphics and Applications, IEEE, vol. 21, issue 3, p. 6-8.

BUJAK, K. R., RADU, I., CATRAMBONE, R., MACINTYRE, B., ZHENG, R., GOLUBSKI, G. (2013). "A psychological perspective on augmented reality in the mathematics classroom" en Computers \& Education, vol. 68, p. 536-544.

CHENG, K. H., TSAI, C. C. (2013). "Affordances of augmented reality in science learning: Suggestions for future research" en Journal of Science Education and Technology, vol. 22, issue 4, p. 449-462.

CRAIG, A. B. (2013). “Augmented reality applications" en Understanding augmented reality p. 221-254.

DEDE, C. (2000). "Emerging influences of information Technology on school curriculum" en Journal of Curriculum Studies, vol. 23, issue 2, p. 282-303.

DONG, S., BEHZADAN, A. H., CHEN, F., KAMAT, V. R., (2013). "Collaborative visualization of engineering processes using tabletop augmented reality" en Advances in Engineering Software, vol. 55, p. 45-55.

DUNLEAVY, M., DEDE, C., MITCHELL, R. (2009). "Affordances and limitations of immersive participatory augmented reality simulations for teaching and learning" en Journal of Science Education and Technology, vol. 18, p. $7-22$.

FONSECA, D., MARTí, N., REDONDO, E., NAVARRO, I., SÁNCHEZ, A. (2014). "Relationship between student profile, tool use, participation, and academin performance with the use of Augmented Reality technology for visualized architecture models" en Computers in Human Behavior, vol. 31, p. 434445.

FRANK, J. A., KAPILA, V. (2017). “Mixed-Reality Learning Environments: Integrating Mobile Interfaces with Laboratory Test-beds" en Computer \& Education, vol. 10, p. 84-104.

GOOGLE (2020). Después de tres años de desarrollo, Google cierra Project Tango para centrarse exclusivamente en ARCore. $<$ https://www.xataka.com/realidad-virtual-aumentada/despues-de-tres-anosde-desarrollo-google-cierra-project-tango-para-centrarse-exclusivamente-en-arcore $>$. [Consulta : 22 de marzo de 2020].

GUILARTE MARTÍN-CALERO, C. (2008). Innovación docente: Docencia y TICS. Valladolid: Universidad de Valladolid.

HOLOLENS (2020). HoloLens 2, la realidad mixta está lista para la empresa. Documentación de Microsoft. < https://www.microsoft.com/es-es/hololens> [Consulta : 22 de marzo de 2020]. 
IBÁÑEZ, M. B., DELGADO-KLOOS, C. (2018). “Augmented reality for STEM learning: A systematic review" en Computers \& Education, vol. 123, p. 109-123.

KAUFMANN, H. (2002). "Construct3D: An Augmented Rality Application for Mathematics and Geometry Education” en Proceedings of ACM Multimedia Conference 2002, p. 656-657.

KAUFMANN, H. (2003). “Collaborative augmented reality in education”. En Imagina 2003 conference. Monaco: Mediax, Monaco.

KREJINS, K., ACKER, F. V., VERMEULEN, M., BUUREN, H. V. (2013). "What stimulates teachers to integrate ICT in their pedagogical practices? The use of dicgital learning materials in education" en Computers in Human Behavior, vol. 29, issue 1, p. 217-225.

LAW, N., PELGRUM, W. J., PLOMP, T. (2008). Pedagogy and ICT use in schools around the world: Findings from the IEA SITES 2006 study. Hong Kong: Springer.

LIDAR (2020) The new iPad Pro's LIDAR sensor is an AR hardware solution in search of software. $<$ https://www.theverge.com/2020/3/19/21185200/apple-ipad-pro-lidar-sensor-ar-hardware-solutionsoftware-apps-augmented-reality>. [Consulta : 22 de marzo de 2020].

MAITI, A., TRIPATHY, B. (2012). "Different platforms for remote laboratories in mobile devices" en International Journal of Modern Education and Computer Science, vol. 4, issue. 5, p. 38-45.

MAGICLEAP (2020). Magic Leap, accelerate remote work. <www.magicleap.com> [Consulta : 22 de marzo de 2020].

MARTÍN GUTIÉRREZ, J., FABIANI, P., BENESOVA, W., MENESES, M. D., MORA, C. E. (2015). "Augmented reality to promote collaborative and autonomous learning in higher education" en Computers in Human Behavior, vol. 51, p. 752-761.

MILGRAM, P., TAKEMURA, H. (1994). "Augmented reality: A class of displays on the realityvirtuality continuum" en Telemanipulator and Telepresence Technologies, vol. 2351, p. 282-292.

NGUYEN, L., BARTON, S., NGUYEN, L. (2015). "iPads in higher education: Hype and hope" en British Journal of Educational Technology, vol. 46, issue 1, p. 190-203.

PAN, Z., CHEOK, A.D., YANG, H., ZHU, J., SHI, J. (2006). "Virtual reality and mixed reality dor virtual learning environments" en Computers and Graphics, vol. 30, issue 1, p. 20-28.

RADU, I. (2014). "Augmented reality in education: A meta-review and cross-media analysis" en Personal and Ubiquitous Computing, vol. 18, issue 6, p. 1533-1543.

ROCA, J., GAGNÉ, M. (2008). "Understanding e-learning continuance intention in the workplace. A self-determination theory perspective" en Computers in Human Behavior, vol. 24, p. 1585 -1604.

SHEN, C. X., LIU, R. D., WANG, D. (2013). "Why are children attracted to Internet? The role of need satisfaction perceived online and perceived in daily real life" en Computers in Human Behaviour, vol. 29, issue 1, p. 185-192.

WILLIAMS, A., PENCE, H. (2011). "Smartphones, a powerful tool in the chemistry classroom" en Journal of Chemical Education, vol. 88, issue 6, p. 683-686.

WU, H. K., LEE, S. W. Y., CHANG, H. Y., LIANG, J. C. (2013). "Current status, opportunities and challenges of augmented reality in education" en Computers \& Education, vol. 62, p. 41-49. 